\title{
Double dissociation between representational personal and extrapersonal neglect
}

\author{
S. Ortigue, PhD; P. Mégevand, MD; F. Perren, MD; T. Landis, MD; and O. Blanke, MD
}

\begin{abstract}
Background: Although perceptual and representational neglect are frequently associated, the demonstration of a double dissociation between both neglect forms suggests that both rely on different central mechanisms. In addition, perceptual neglect can be selectively observed within personal space or extrapersonal space. However, it is not known whether the latter dissociation also exists in representational neglect. Methods: The authors investigated this question in two brain-damaged patients with anatomically different lesions sites, using neuropsychological tests specifically designed to assess perceptual and representational neglect in both personal and extrapersonal space. Results: Patients presented a double dissociation with respect to personal and extrapersonal space in representational neglect. Conclusions: These data suggest that the cerebral networks that process mental space representation use similar principles of space compartmentalization as those used by cerebral networks processing perceived space.
\end{abstract}

NEUROLOGY 2006;66:1414-1417

Patients with unilateral neglect fail to attend to or to explore the space contralateral to their brain damage in the absence of any primary sensory deficits. Unilateral neglect exists in response to sensory stimuli (perceptual neglect), but also in the absence of such sensory input, by imagining familiar spatial scenes (representational neglect). ${ }^{1,2}$ Although these two forms of unilateral neglect are frequently associat$\mathrm{ed},{ }^{1}$ they may occur independently. A few cases of "pure" representational neglect have been described $^{2,3}$ corresponding to the inability to describe from memory the contralesional part of a familiar environment, independently of the imposed imagined viewpoint.

Within the perceptual domain, space may be subdivided into different spatial compartments, such as personal space (one's own body) and extrapersonal space, which comprises the near space (within reaching distance) and far space (beyond reaching distance). ${ }^{4}$ Whereas it has been demonstrated for perceptual neglect that personal and extrapersonal space can be selectively affected, ${ }^{5-7}$ patients with representational neglect have only been investigated with respect to spatial imagery in extrapersonal space using familiar city squares, familiar room interiors, or country maps. ${ }^{2}$ Yet, it is not known whether a dissociation with respect to personal and extrapersonal space also exists in representational neglect. This is relevant for the question of whether percep- tual and mental spatial cognition rely on common or separate organizational principles., ${ }^{2,3}$ We thus systematically assessed neglect patients with a battery of tests specifically designed to test representational neglect and perceptual neglect for personal and extrapersonal space. Here, we describe two patients: one with representational neglect for personal, but not extrapersonal space, and another patient with the opposite pattern.

Case reports. Patient 1. A 67-year-old, right-handed baker initially had a left-sided weakness and left hemihypoesthesia, which partially recovered within 1 week. There was no visual field deficit and no visual extinction. A detailed neuropsychological assessment including standard comprehension, naming, reading, writing, phonologic, and semantic verbal fluency tasks did not show any sign of aphasia. There was no deficit in verbal long-term memory (Rey Auditory-Verbal Test). A mild dysexecutive deficit was observed (Stroop, Luria Three-Step Task). Apart from the spatial domain, mental imagery was flawless (drawing and verbal descriptions from memory of objects and animals). MRI showed a small lesion restricted to anterior part of the posterior limb of the internal capsule in the territory of the anterior choroidal artery (figure 1).

Patient 2. A 66-year-old, right-handed police officer had several short-lasting episodes of left hemisensory loss and left hemiataxia. At admission he had a left hemianopia, a left spatial hemineglect, a left-sided weakness, and left hemihypoesthesia. There was no tactile or auditory extinction. Detailed neuropsychological assessment including standard comprehension, naming, reading, writing, phonologic, and semantic verbal fluency tasks did not show any sign of aphasia. There was no deficit in verbal long-term memory (Rey Auditory-Verbal Test) and no dysexecutive disorder. However, there was a left perceptual and represen-

From Functional Brain Mapping Laboratory (S.O.), University Hospital of Geneva, and Department of Fundamental Neurosciences, University Central Medical School, Geneva, Department of Neurology (S.O., P.M., F.P., T.L., O.B.), University Hospital of Geneva, and Laboratory of Cognitive Neuroscience (O.B.), Brain Mind Institute, Ecole Polytechnique Fédérale de Lausanne, Switzerland.

Supported by the Swiss National Science Foundation (3100-065096.01/2 and 3100-0650232.02; 3100-067874.02) and the Swiss Foundation for Biology and Medicine (1223/PASMA 111563/1)

Disclosure: The authors report no conflicts of interest.

Received September 7, 2005. Accepted in final form January 23, 2006.

Address correspondence and reprint requests to Dr. S. Ortigue, Dartmouth Functional Brain Imaging Center, Center for Cognitive Neuroscience, Department of Psychology and Brain Sciences, Dartmouth College, 6162 Moore Hall, Rm. 432, Hanover, NH 03755; e-mail: stephanie.ortigue@dartmouth.edu 

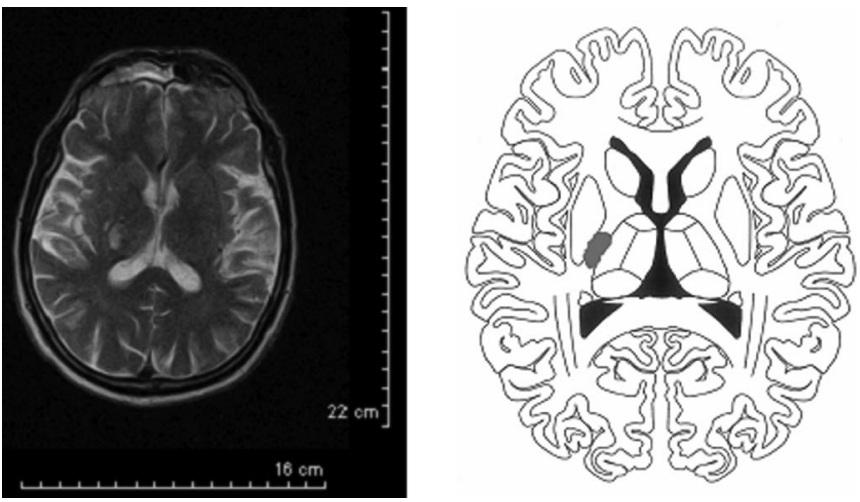

Figure 1. The MR images of these patients were mapped onto modified templates. Patient 1. MRI and template of the brain showing a small ischemic lesion of the posterior limb of the internal capsule in the territory of the right anterior choroidal artery. ${ }^{16}$

tational spatial hemineglect, spatial dysgraphia (a tendency to write predominantly on the right side of a sheet of paper, to produce wandering lines with overlapping words and letters, and to split words incorrectly), ${ }^{15}$ and spatial dyslexia (misreading text appearing on the contralesional side of space, skipping from line to line in reading written text, although reading individual words was preserved)

Within 1 week, the left hemianopia regressed into an upper left quadranopia and the left perceptual hemineglect, the spatial dysgraphia, and dyslexia disappeared. Only the left representational visuospatial hemineglect persisted. MRI showed a rightsided lesion in the territory of the posterior cerebral artery, involving the inferolateral thalamus, the splenium of the corpus callosum, and parts of the medial temporal and occipital lobes (figure 2).

Methods. Perceptual and representational neglect were tested in both personal and extrapersonal space.

Perceptual neglect. Perceptual extrapersonal space. To examine neglect in extrapersonal space, ${ }^{8}$ different tasks were given in near and far extrapersonal space. ${ }^{9}$ Assessment of these two compartments is of particular interest, especially as a growing body of evidence in functional neuroimaging has demonstrated anatomic differences in healthy subjects between performing perceptual and motor tasks in near and far space..$^{10,11}$ In the current study, near extrapersonal space was assessed using the Line Bisection Task and the Bell Cancellation Task. ${ }^{8,9}$ The percentage mean deviation was calculated for the Line Bisection Task. Misses were scored for the Bell Cancellation Task (total of 35 items; 15 right, 15 left, 5 middle). Far extrapersonal space was assessed using the Line Bisection Task ${ }^{9}$ (Patient 1) and a verbal description (Patient 2) of a distant visual scene.

Perceptual personal space. Patients were asked to reach for their left hand with the right hand, with eyes opened. ${ }^{5} \mathrm{~A}$ four-level scale was used $(0$, normal performance; 1 , the target is reached with hesitation and is searched; 2 , searching is stopped before the target can be reached; 3 , no attempt to reach the target).

We also carried out the Fluff Test ${ }^{7}$ and the Reformulated Comb Test..$^{7,12}$

The Fluff Test required the patients to reach with their right hand for 15 targets (2-cm diameter) that were attached to their body. Three targets were attached to the right side and three to the left side of the torso (with respect to the frontal midline). Three targets were attached to the right leg, three to the left leg, and three to the left arm. No targets were placed on the right arm because the task was performed with the right hand. Each patient was blindfolded and asked to remove all targets.

In the Reformulated Comb Test, each patient was asked to comb his hair for 30 seconds with the right hand. The number of strokes applied to the right and left side of the head, as well as ambiguous strokes, were measured to calculate the following score $^{7}: x_{\text {comb }}=([$ left - right strokes $] /[$ left + ambiguous + right
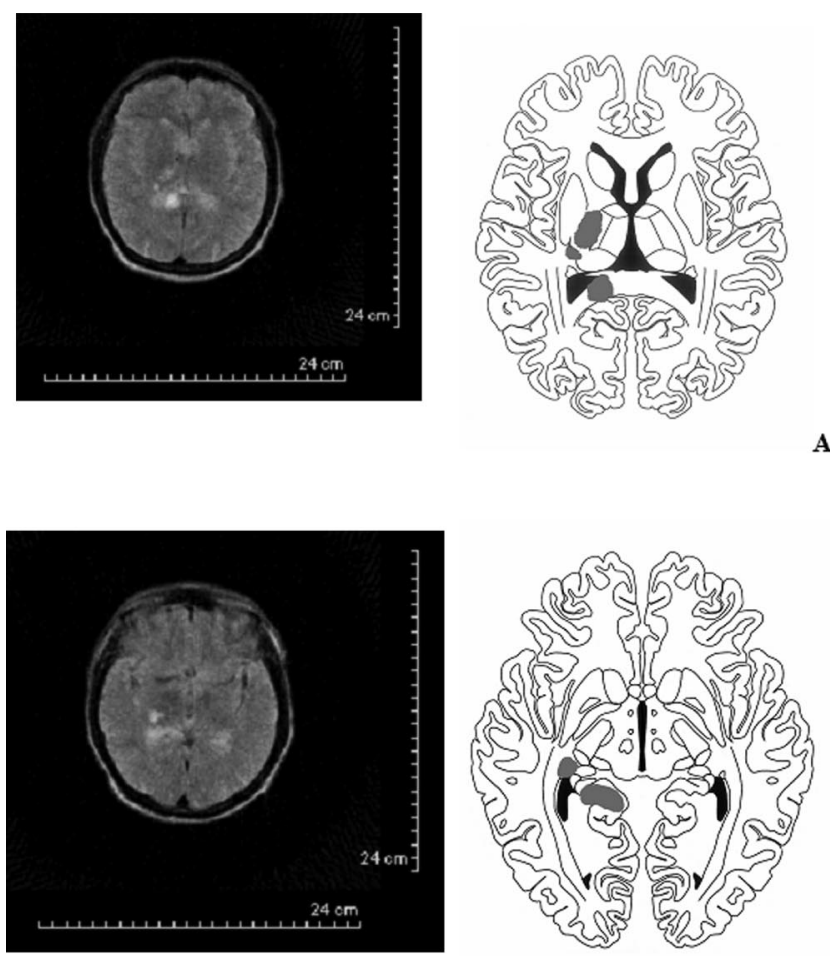

B
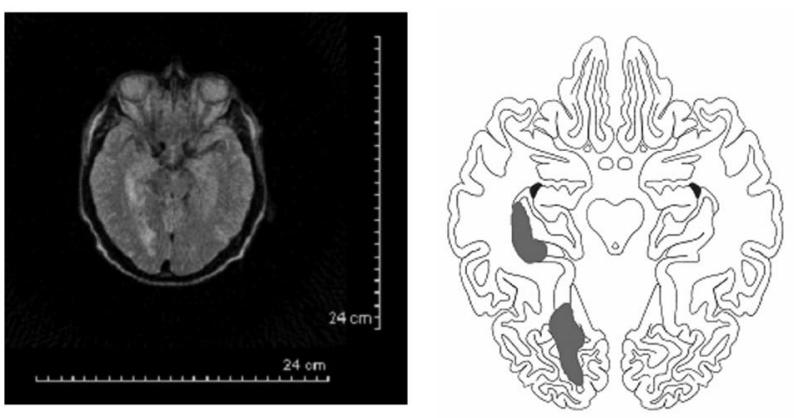

C

Figure 2. Patient 2. MRI and templates of the brain showing ischemic embolic lesions in the territory of the right posterior cerebral artery. (A) Lesion of the inferolateral part of the thalamus impinging upon the posterior limb of the internal capsule (thalamogeniculate branch of P2) and a lesion of the splenium of the corpus callosum. (B, C) Medial temporal and occipital lesions.

strokes]) * 100. This value yields a score between $-100 \%$ (total left neglect) and $+100 \%$ (total right neglect).

Representational neglect. Representational extrapersonal space. Each patient was asked to mentally visualize a familiar square (the Place Neuve in Geneva; local version of the Piazza del Duomo). ${ }^{1,2}$ This is a well-known place in Geneva with many wellknown buildings (figure 3). Each patient was asked to imagine this square from two predefined opposing viewpoints (by $\left.180^{\circ}\right) .^{1-3}$ These were at the entrance of the Parc des Bastions and at the Théâtre de Genève. Each patient's response, which was given from one of these two viewpoints, served as a control for the other viewpoint on the basis that a right-sided response from a viewpoint automatically becomes a left-sided response from its $180^{\circ}$ opposite viewpoint. In this sense, global numbers of reported items might be different from one patient to another. Five normal control subjects (mean age $72 \pm 4$ years) performed this task. In average, they reported 6.8 left-sided items and 7.1 right-sided items. Their results showed no difference between the reported left-sided items and the right-sided items $(p=0.49)$. 


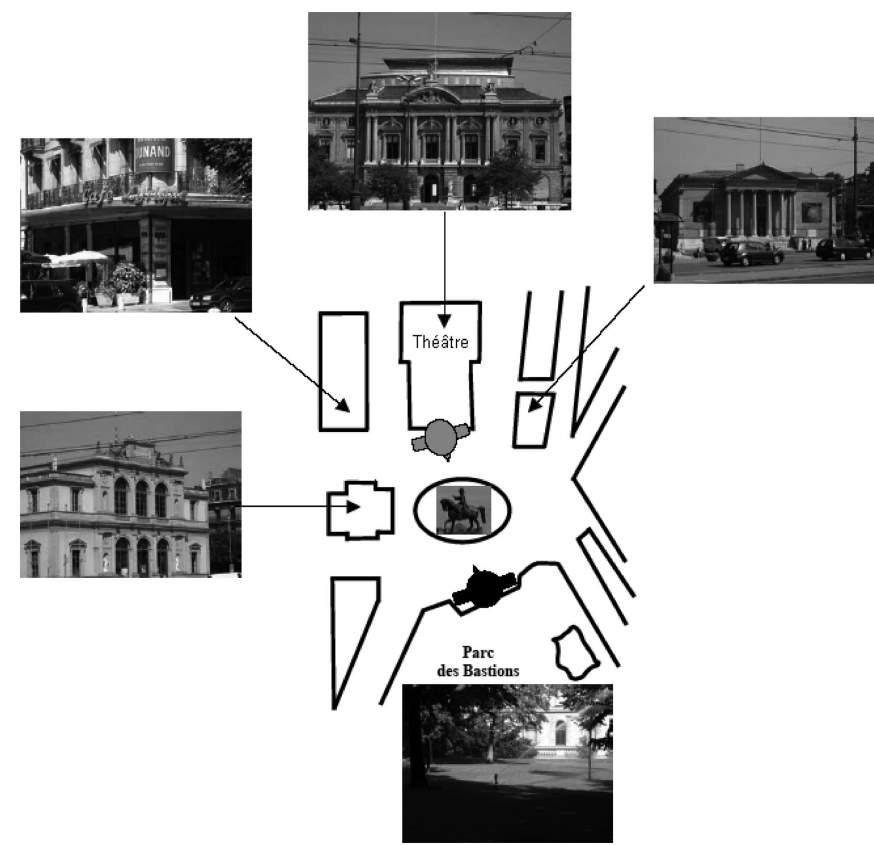

Figure 3. The Place Neuve in Geneva with its principal architectonic elements.

Representational personal space. We developed three tasks for assessing the patients' performance in representational personal space: the Modified Fluff Test, the Modified Comb Test, and the Modified Razor Test.

The Modified Fluff Test required the patients to indicate, from memory and with eyes closed, the location of 12 targets $(2-\mathrm{cm}$ diameter) that were previously (5-minute delay) attached to their body. Three targets were attached to the right side and three to the left side of the torso (with respect to the frontal midline). One target was attached to the right leg, one to the left leg, and four to the left arm. No targets were placed on the right arm because, as in the original Fluff Test, the task was performed with the right hand. This modified version of the Fluff Test is considered to assess the representational domain on the basis of the instruction, which asks the patients to indicate from memory the previous location of each target after they were removed by the examiner. In the original version of the Fluff Test, the patients were asked to reach each target while it was still on the patients' bodies.

In the Modified Comb Test, each patient was asked to simulate combing his hair for 30 seconds with the right hand, while he was blindfolded. As in the original Comb Test, the number of strokes applied to the right and left side of the head, as well as ambiguous strokes, were measured to calculate the following score: $x_{\text {comb }}=$ (left - right strokes)/(left + ambiguous + right strokes). This value yields a score between -1 (total left neglect) and +1 (total right neglect).

In the Modified Razor Test, ${ }^{12}$ each patient was instructed to simulate shaving himself for 30 seconds with the right hand, while he was blindfolded. As in the initial Razor Test, the number of strokes applied to the right and left side of the head, as well as ambiguous strokes, were measured to calculate the following score: $x_{\text {razor }}=($ left - right strokes $) /($ left + ambiguous + right strokes). This value yields a score between -1 (total left neglect) and +1 (total right neglect).

Results. Perceptual neglect. Perceptual extrapersonal space. Patient 1. In near space, the Line Bisection Task (mean of deviation of $+5 \%$ ) and the Bell Cancellation Task (35/35 correct) were flawless. No far extrapersonal neglect was observed (line bisection: $+4 \%$ of mean deviation). Moreover, no reaction time difference was observed between the left $(521 \pm 126$ milliseconds) and right (561 \pm 174 milliseconds) hemispace during a classic target detection paradigm (from the Test of Everyday Attention [TEA] battery) realized 12 days after hospitalization. Patient 2 . No near extrapersonal neglect was observed in the Line Bisection Task (percent of mean deviation: $+7 \%$ ) or the Bell Cancellation Task (34/35 correct). The description of a far distant visual scene was flawless (no far extrapersonal neglect).

Perceptual personal space. Both patients performed well in the Fluff Test (15/15 items detected) as well as in the Reformulated Comb Test $\left(x_{\text {comb }}\right.$ for Patient $1=$ $-12.5 \% ; x_{\mathrm{comb}}$ for Patient $2=-6 \%$ ).

Representational neglect. Representational extrapersonal space. Patient 1. Familiar square description did not exhibit any significant signs of hemineglect (4/5 on the left side from the first viewpoint and 4/6 on the left side from the $180^{\circ}$ opposite viewpoint). All items on the right side were correctly reported from both viewpoints. Patient 2. Familiar square description led to a strong left representational hemineglect. The patient reported only 4 of 10 items on the left side from the first viewpoint and 5 of 13 items on the left side from the $180^{\circ}$ opposite viewpoint. All items on the right side were correctly reported from both viewpoints.

Representational personal space. Patient 1. The Modified Fluff Test revealed a mild left representational personal neglect, which was characterized by two systematic left-sided omissions in the upper body. All items on the right side were correctly reported. The Modified Comb Test revealed a strong left representational personal neglect characterized by a rightward deviation bias of $+69 \%$ and $+60 \%$. The Modified Razor Test also revealed a strong left representational personal neglect characterized by a rightward deviation bias of $+46 \%$ and $+29 \%$. Patient 2 . This patient showed no representational personal neglect. The Modified Fluff Test (12/12 items detected) and the Modified Comb Test (mean $x_{\text {comb }}$ deviation $=-14 \%$ ) were both within normal range.

Discussion. We investigated whether the compartments of personal and extrapersonal space as observed in perceptual space could also be demonstrated in imagined space. We systematically searched for this dissociation and report here about two patients without perceptual neglect, but with selective representational neglect either for personal space (Patient 1) or for extrapersonal space (Patient 2). From a purely behavioral viewpoint, this double dissociation suggests that mental imagery relies on the same or similar processing principles with respect to personal and extrapersonal space as perception does. It could be argued that the current dissociation might be due to involved motor mechanisms as our tests for personal representational neglect depended on manual motor responses, whereas our tests for extrapersonal representational neglect depended on verbal motor responses. Yet, in both patients, there were no signs of perceptual personal neglect that was tested with the same manual motor responses as the tests for representational personal neglect that were pathologic in Patient 1 . If representational neglect were indeed related to motor mechanisms, one could postulate that this motor difference applied only for targets in representational 
space. Another difference between tests for personal and extrapersonal representational neglect is that tests for the former space relied on procedural (Comb and Razor Tests) and short-term memory (Fluff Test), whereas our tests for the latter space relied on long-term memory. Although our results within representational personal space (in Patient 1) show that neglect was found independently of the involved memory mechanisms (procedural vs short term) and that memory as examined by the neuropsychological examination was normal in both patients, we cannot exclude that memory-related differences between personal and extrapersonal tests might have influenced our results.

The idea underlying the principle of double dissociation would, however, suggest that for our tasks, different, though maybe overlapping, structures or networks were used. Ideally, functional double dissociations should go along with clearly dissociated anatomic lesion sites. However, especially with cortical lesions, many functionally dissociated neglect syndromes have shown large overlapping lesion. In the current two cases, the lesions do not overlap, which may allow some anatomicofunctional speculations.

The first case with a "pure" representational personal neglect is quite unique for two reasons: 1) To our best knowledge, no case has yet been described with "pure" personal representational neglect (as defined with the current tests), and 2) the lesion leading to this defect is very small, restricted to the anterior part of the posterior limb of the internal capsule in the territory of the anterior choroidal artery. Infarcts in the posterior limb of the internal capsule are usually larger and may lead to severe multimodal persistent neglect, ${ }^{13}$ particularly for visually guided movements, ${ }^{14}$ suggesting a disruption of connections between the pulvinar and the parietal lobe. In our case, the visual thalamocortical transcapsular connections, producing extrapersonal neglect, have very probably been left intact. We speculate that this might have led to a pure personal neglect only in the representational domain, as with opened eyes, personal space was unimpaired.

Our second case had several lesions in the territory of the right posterior cerebral artery. Most prob- ably, the large inferolateral thalamic lesion was responsible for the "pure" representational neglect of extrapersonal space, as this kind of neglect has already once been reported with an isolated similarly placed thalamic lesion. ${ }^{2}$ In this second case, visual pulvinar-parietal connections were probably interrupted, whereas more anterior somatosensory thalamoparietal connections were left intact. It is noteworthy that in both cases, the lesions do not touch upon the classic lesion site for perceptual neglect, which is the posterior lateral temporoparietal junction. Our observation stresses the importance of discrete subcortical lesions for the integrity of space representation.

\section{Acknowledgment}

The authors thank the patients for their participation.

\section{References}

1. Bisiach E, Luzzatti C. Unilateral neglect of representational space. Cortex 1978;14:129-133.

2. Ortigue S, Viaud-Delmon I, Annoni JM, et al. Pure representational neglect after right thalamic lesion. Ann Neurol 2001;50:401-404.

3. Ortigue S, Viaud-Delmon I, Michel C, et al. Pure imagery hemi-neglect of far space. Neurology 2003;60:2000-2002

4. Grüsser OJ, Landis T. In: Cronly-Dillon JR, ed. Visual agnosias and other disturbances of visual perception and cognition, vol. 12. London: Macmillan, 1991

5. Bisiach E, Perani D, Vallar G, Berti A. Unilateral neglect: personal and extra-personal. Neuropsychologia 1986;24:759-767.

6. Guariglia C, Antonucci G. Personal and extrapersonal space: a case of neglect dissociation. Neuropsychologia 1992;30:1001-1009.

7. Beschin N, Robertson IH. Personal versus extrapersonal neglect: a group study of their dissociation using a reliable clinical test. Cortex 1997;33:379-384

8. Halligan PW, Marschall JC. Left neglect for near but not for far space in man. Nature 1991;350:498-500.

9. Vuilleumier P, Valenza N, Mayer E, et al. Near and far visual space in unilateral neglect. Ann Neurol 1998;43:406-410.

10. Fink GR, Marshall JC, Shah NJ, et al. Line bisection judgments implicate right parietal cortex and cerebellum as assessed by fMRI. Neurology $2000 ; 54: 1324-1331$.

11. Weiss PH, Marshall JC, Zilles K, Fink GR. Are action and perception in near and far space additive or interactive factors? Neuroimage 2003;18: 837-846.

12. McIntosh RD, Brodie EE, Beschin N, Robertson IH. Improving the clinical diagnosis of personal neglect: a reformulated comb and razor test. Cortex 2000;36:289-292.

13. Cambier J, Graveleau Ph, Decroix JP, Elghozi D, Masson M. Anterior choroidal artery syndrome. Neuropsychological study of 4 cases. Rev Neurol 1983;139:553-559.

14. Ferro JM, Kertesz A. Posterior internal capsule infarction associated with neglect. Arch Neurol 1984;41:422-424.

15. Ortigue $\mathrm{S}$, Landis T. Letters lost in space: hemispace dependent handwriting errors. Neuroreport 2004;15:2545-2548.

16. Tatu L, Moulin T, Bogousslavsky J, Duvernoy H. Arterial territories of the human brain: cerebral hemispheres. Neurology 1998;50:1699-1708. 